\title{
Sialolitíase submandibular em paciente HIV-positivo fazendo uso de antirretrovirais: relato de caso
}

Lucas Teixeira BRITO'; Vinicius Marques OLIVEIRA'; Laiz Moreira de PAULA'; Tiago Oliveira TAVARES²; Ítalo Cordeiro TOLEDO²

1 - Cirurgião-Dentista, Residente em Cirurgia e Traumatologia Buco-maxilo-facial pelo Hospital Estadual de Urgências da Região Noroeste Governador Otávio Lage de Siqueira (HUGOL); 2 - Cirurgião-Dentista, Mestre, Especialista e Preceptor da Residência em Cirurgia e Traumatologia Buco-maxilo-facial pelo Hospital Estadual de Urgências da Região Noroeste Governador Otávio Lage de Siqueira (HUGOL).

\section{Resumo}

Sialolitíase é uma alteração obstrutiva de glândula salivar relativamente comum e de patogênese incerta. Desde o início da era HAART, estudos têm levantado hipóteses a respeito do aumento da prevalência de cálculos em diversos locais do organismo, em pacientes infectados pelo HIV. O presente artigo relata um caso clínico da possível associação entre a formação de cálculo salivar em paciente diagnosticado com o HIV e em tratamento por meio de antirretrovirais.

PALAVRAS-CHAVE: Cálculos das Glândulas Salivares; HIV; Antirretrovirais; Terapia Antirretroviral de Alta Atividade. 


\section{Introdução}

Doenças obstrutivas de glândulas salivares são alterações não neoplásicas que podem ser causadas por variações anatômicas, estenose dos ductos, cálculos, corpos estranhos, alterações inflamatórias entre outros. A sialolitíase é a principal causa da obstrução das glândulas salivares'. Sua patogênese é incerta, e pode estar relacionada à estrutura da glândula envolvida e de seu ducto e à composição salivar ${ }^{2}$. No contexto do tratamento, a decisão se baseia na presença de sinais e sintomas, na localização, tamanho e quantidade dos cálculos salivares ${ }^{3}$.

O vírus da imunodeficiência humana (HIV) é o agente etiológico da Síndrome da Imunodeficiência Adquirida (AIDS), doença devastadora caracterizada por apresentar um grande desafio à área da saúde. A AIDS é frequentemente relacionada a diversas doenças nas glândulas salivares, podendo estar associada ao aparecimento de cálculos salivares, devido à sua capacidade de redução do fluxo salivar e alteração da composição química da saliva

Com a adoção da terapia antirretroviral altamente eficaz (HAART) para controle da replicação do vírus e da supressão imunológica, o paciente infectado pelo HIV tem apresentado melhor prognóstico e um aumento em sua qualidade de vida. A incidência de manifestações orais diminuiu com o advento dessa terapia, mas a formação de cálculos em diversos locais do organismo tem sido apresentada na literatura e relacionada ao uso dos antirretrovirais ${ }^{5}$.

Diante dessa realidade, esse artigo tem como objetivo relatar um caso clínico da possível associação entre a formação de cálculo salivar em paciente diagnosticado com o HIV e em uso de antirretrovirais.

\section{Relato do caso}

Paciente do gênero masculino, 56 anos de idade, melanoderma, compareceu ao serviço de Cirurgia e Traumatologia 
Bucomaxilofacial de um Hospital de Urgência, com queixa de episódios recorrentes de aumento de volume em região mandibular em lado esquerdo com três anos de duração, associados a dor, diminuição do fluxo salivar e drenagem de secreção purulenta em cavidade oral.

O paciente afirmou ser infectado por HIV, estar em acompanhante médico devido à sua comorbidade crônica e fazer uso regular de medicação antirretroviral composto por Tenofovir Disoproxil Fumarato/Lamivudina/Efavirenz (300mg/300m$\mathrm{g} / 600 \mathrm{mg}$ ), um comprimido por dia, há mais de 05 anos. Em nenhum momento apresentou e relatou qualquer evento relacionado à AIDS.

Ao exame físico, notou assimetria em região submandibular esquerda à custa de um aumento de volume nodular de consistência endurecida, imóvel e com queixas álgicas à manipulação. Mediante ordenha, não foi observado saída de secreção purulenta em cavidade oral e durante a palpação da região de assoalho bucal em região posterior ipsilateral também foi possível sentir a lesão de aspecto nodular.

O exame de ultrassonografia da região cervical esquerda apresentou imagens sugestivas de aumento de dimensões em glândula submandibular esquerda associada à ecotextura levemente heterogênea. Observou-se ainda fluxo vascular aumentado, destacando-se a presença de cálculo localizado no interior da porção extra glandular do ducto principal, com hipótese diagnóstica de submandibulite e sialolitíase (Figura 1). Para melhor avaliação e definição de conduta, foi solicitado o exame de tomografia computadorizada de face (feixe em leque), que concluiu imagens que sugeriam heterogeneidade e aumento das dimensões da glândula submandibular esquerda, cálculo salivar hiperdenso, medindo cerca de 23 milímetros, sugerindo processo inflamatório correspondente com sialoadenite (Figura $2 \mathrm{~A}-\mathrm{B}$ ). 


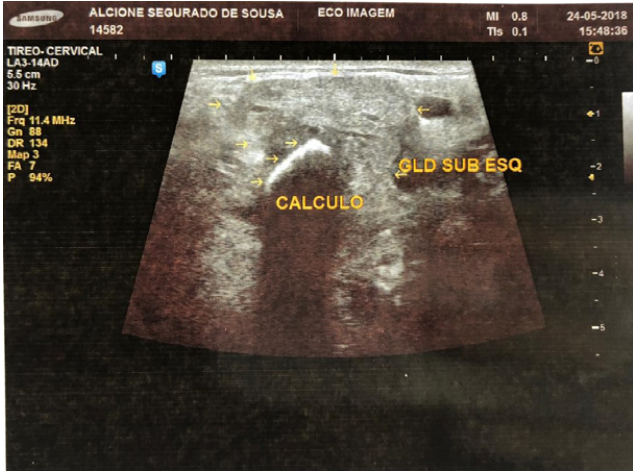

FIGURA 1 - Imagem obtida pelo exame de ultrassonagrafia da região cervical sugerindo a presença de cálculo salivar nas proximidades da glândula submandibular esquerda

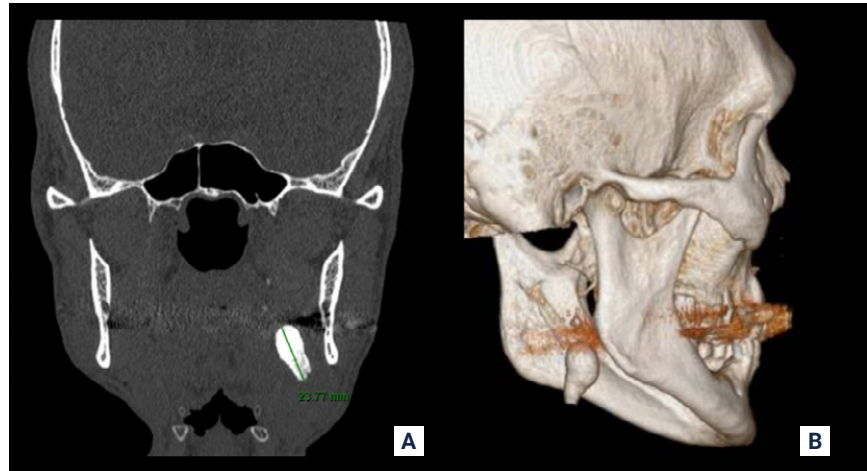

FIGURA 2 - (A) Tomografia computadorizada em reconstrução multiplanar coronal apresentando imagem hiperdensa em região de glândula salivar esquerda. (B) Reconstrução 3D para avaliação tridimensional do cálculo salivar

Após avaliação dos exames de imagem e associação dos mesmos com o exame clínico, foi optada, juntamente com o paciente, pela remoção do cálculo salivar por meio de acesso cirúrgico direto. $\mathrm{O}$ paciente foi submetido à avaliação cardiológica que concluiu baixo risco cirúrgico. Os exames laboratoriais solicitados estavam dentro do padrão de normalidade.

A abordagem cirúrgica para excisão do cálculo salivar foi realizada em centro cirúrgico sob anestesia geral balanceada e intubação nasotraqueal. Para melhor exposição da glândula e avaliação estrutural da mesma, o acesso cirúrgico de escolha foi o acesso de Risdon (submandibular). Ao expor a glândula submandibular, foi possível notar um bom aspecto da mesma, portanto, optou-se por evitar a remoção da glândula afetada. Foi realizado divulsão romba através da glândula para localizar o cálculo salivar. Se fez necessário realizar pressão digital intraoral no sentido ínfero-lateral para melhor divulsão dos tecidos que envolviam o cálculo, bem como, para facilitar sua a preensão e remoção do mesmo. Após excisão do sialolito, observou-se, mediante ordenha, a patência satisfatória do ducto glândular. O fechamento do acesso cirúrgico foi realizado com sutura dos planos teciduais com fios Vicryl 4-0 e Nylon 5-0. O material removido no transcirúrgico foi encaminhado ao exame histopatológico para confirmar o diagnóstico (Figura 3). 


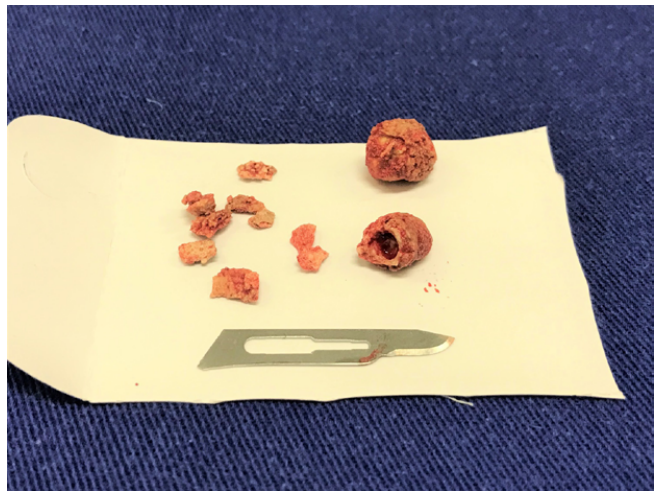

FIGURA 3 - Cálculo salivar após exérese

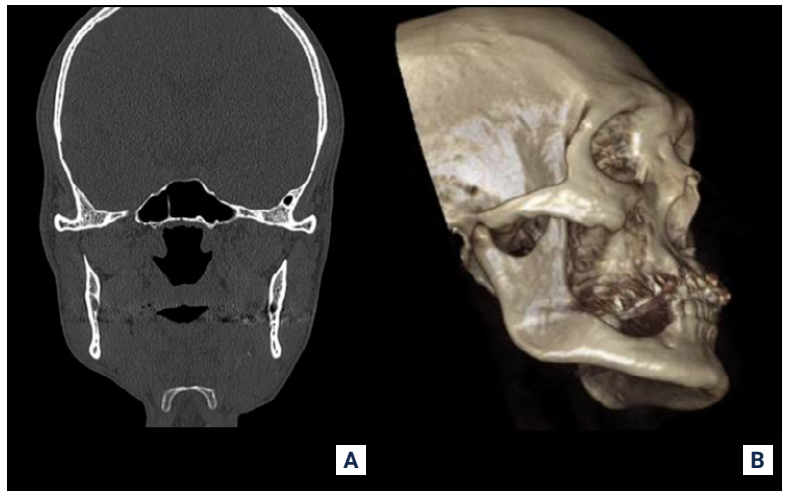

FIGURA 4 - (A) Tomografia computadorizada pós-operatória em reconstrução multiplanar coronal apresentando imagem que sugere remoção total de cálculo salivar. (B) Reconstrução 3D pós-operatória

O exame imaginológico pós-operatório sugeriu completa remoção do cálculo salivar (Figura 4 A-B). Transcorridos 07 meses de acompanhamento ambulatorial, o paciente apresentou bom aspecto cicatricial do acesso cirúrgico, livre de processos inflamatórios e/ou infecciosos. O mesmo afirmou ter findado os episódios recorrentes de aumento de volume, melhora do fluxo salivar e ausência de alterações sensitivas e motoras. O resultado do exame histopatológico confirmou hipótese diagnóstica de cálculo salivar.

\section{Discussão}

A HAART tem se mostrado altamente eficaz aos pacientes infectados pelo HIV, proporcionando a redução da replicação viral, o aumento do número de linfócitos TCD4+ e a restauração da imunidade do paciente infectado ${ }^{6}$. Em 2014, o Ministério da Saúde do Brasil iniciou a oferta da HAART por meio de uma nova terapia antirretroviral de primeira linha composta pela combinação de três medicamentos em um comprimido: Tenofovir (300mg), Lamivudina (300mg) e Efavirenz (600mg). As duas primeiras medicações são inibidores da transcriptase reversa análogos de nucleosídeos e a terceira é um inibidor da transcriptase reversa não análogos de nucleosídeos ${ }^{7,8}$. Essa nova combinação propõe uma melhor adesão ao tratamento e a diminuição de 
efeitos colaterais. O paciente do caso clínico mantém o uso dessa medicação há 05 anos, e não foram encontradas falhas no tratamento proposto, durante o período de acompanhamento médico.

Alguns medicamentos antirretrovirais, como o Atazanavir/ritonavir e o indinavir foram apontados na literatura como precursores de cálculos em diversas localidades no corpo humano, como nos rins, no fígado, na bexiga, na uretra e até mesmo, nas glandulares salivares ${ }^{9-12}$. Em grande parte dos estudos presentes na literatura, os pacientes fizeram uso de outros diversos antirretrovirais e suas associações, portanto os mesmos também devem ser considerados nos casos de litíases. Os fármacos utilizados pelo paciente durante o período de 05 anos foram citados nos artigos que envolvem o aparecimento dos cálculos. Devido à existência de outros esquemas terapêuticos propostos para o controle da AIDS, são necessários novos estudos clínicos sobre as combinações utilizadas recentemente e a sua relação com a formação de cálculos.

A diminuição da função das glândulas salivares, resultando em xerostomia, é uma condição clínica frequentemente encontrada em pacientes contaminados pelo HIV e está diretamente associada à diminuição da quantidade das células TCD4+. Com o início da era HAART, alguns estudos demostraram que essa terapia também pode estar associada à redução do fluxo salivar e hipertrofia das glândulas salivares ${ }^{5,13}$, portanto, a associação desses dois fatores deve ser fortemente considerada ao aparecimento de cálculos salivares ${ }^{14}$.

A glândula submandibular é a glândula salivar mais acometida pela sialolitíase. A localização de seu ducto (Wharton), sua forma anatômica tortuosa, seu $\mathrm{pH}$ alcalino e a gravidade podem favorecer infecções retrógradas e a precipitação dos sais de cálcio ${ }^{15}$. A obstrução do ducto, tamanho do cálculo, sintomas recorrentes e a presença de processo infeccioso foram os quesitos decisórios para a remoção do cálculo salivar do presente caso clínico. 
Há inúmeros tratamentos comprovadamente eficazes para resolução da sialolitíase, como as abordagens cirúrgica ou endoscópica, sialolitotripsia por ondas de choque, expulsão espontânea com uso de agentes sialagogos e com o uso de laser. Cabe ao cirurgião responsável realizar um planejamento criterioso e individualizado de cada caso, levando em consideração as proporções e localização do cálculo salivar, recursos disponíveis e limitações de cada técnica, com intuito de oferecer o tratamento mais eficaz e assertivo possível. Dentre os tipos de tratamento existentes, o acesso cirúrgico extraoral de Risdon foi o escolhido para o caso relatado, já que os recursos necessários para realização de outras técnicas menos invasivas não estavam disponíveis no hospital. Esse acesso cirúrgico ainda é bastante utilizado em procedimentos que necessitam de acesso à glândula submandibular e permite a excisão completa dos fragmentos calcificados aderidos aos tecidos glandulares ${ }^{16}$.

\section{Conclusões}

A AIDS é uma doença crônica e altamente destrutiva, portanto cada vez mais pacientes estão buscando tratamentos para seu controle, em prol do aumento em sua sobrevida. O uso de antirretrovirais e a presença da infecção pelo HIV são fatores potenciais para o aparecimento de cálculos em diversos locais do organismo. A sialolitíase ainda é pouco descrita e incluída nessa associação, portanto são necessários mais estudos para consolidação dessa hipótese, para que os pacientes HIV-positivos sejam alertados e orientados sobre o aparecimento dessa alteração e para a evolução e melhoria das terapias antirretrovirais.

\section{Referências}

1 - Atienza G, López-Cedrún JL. Management of obstructive salivary disorders by sialendoscopy: a systematic review. Br J Oral Maxillofac Surg. 2015; 53(6): 507-19. doi: 10.1016/j.bjoms.2015.02.024

2 - Avishai G, Ben-Zvi Y, Ghanaiem O, Chaushu G, Gilat H. Sialolithiasisdo early diagnosis and removal minimize post-operative morbidity? Medicina (Kaunas). 2020; 56(7): 332. doi: 10.3390/medicina56070332 
3- Pachisia S, Mandal G, Sahu S, Ghosh S. Submandibular sialolithiasis: A series of three case reports with review of literature. Clin Pract. 2019; 9(1):1119. doi: 10.4081/cp.2019.1119

4 - Capaccio P, Monforte Ad, Moroni M, Ottaviani F. Salivary stone lithotripsy in the HIV patient. Oral Surg Oral Med Oral Pathol Oral Radiol Endod. 2002; 93(5): 525-7. doi: 10.1067/moe.2002.122343

5 - Meer S. Human immunodeficiency virus and salivary gland pathology: an update. Oral Surg Oral Med Oral Pathol Oral Radiol. 2019; 128(1): 52-59. doi: 10.1016/j.0ooo.2019.01.001

6 - Brojan LEF, Marca LM, Dias FA, Rattmann YD. Antiretroviral drug use by individuals living with HIV/AIDS and compliance with the Clinical Protocol and Therapy Guidelines. Einstein (Sao Paulo). 2020; 18:eAO4995. doi: 10.31744/einstein_journal/2020AO4995

7 - Ministério da Saúde. Secretaria de Vigilância em Saúde. Departamento de DST, Aids e Hepatites Virais. Protocolo clínico e diretrizes terapêuticas para manejo da infecção pelo hiv em adultos. Brasília: Ministério da Saúde; 2013.

8 - Nunes-Junior S, Ciosak S. Terapia antirretroviral para hiv/aids: o estado da arte. Revista de Enfermagem UFPE on line [Internet]. 2018; 12(4): 1103-1111. doi: 10.5205/1981-8963-v12i4a231267p1103-1111-2018

9- Lin KY, Liao SH, Liu WC, Cheng A, Lin SW, Chang SY, Tsai MS, Kuo CH, Wu MR, Wang HP, Hung CC, Chang SC. Cholelithiasis and nephrolithiasis in HIV-positive patients in the era of combination antiretroviral therapy. PLoS One. 2015; 10(9):e0137660. doi: 10.1371/ journal.pone. 0137660

10 - Lê MP, Stitou H, Soulie C, Katlama C, Peytavin G. Sialolithiasis in an HIV-1-infected patient treated with atazanavir/ritonavir monotherapy. J Antimicrob Chemother. 2013; 68(3): 727-9. doi: 10.1093/jac/dks433

11 - Pastori D, Esposito A, Cagliuso M, Conti V, Mezzaroma I. Lithiasis of semicircular canals and parotid glands: unusual stones deposition in atazanavir-treated individuals. AIDS. 2009; 23(16): 2233-4. doi: 10.1097/ QAD.0b013e32833147d9

12 - Doco-Lecompte T, Garrec A, Thomas L, Trechot P, May T, Rabaud C. Lopinavir-ritonavir (Kaletra) and lithiasis: seven cases. AIDS. 2004; 18(4): 705-6. doi: 10.1097/00002030-200403050-00022.

13 - Navazesh M, Mulligan R, Karim R, Mack WJ, Ram S, Seirawan H, Greenspan J, Greenspan D, Phelan J, Alves M; Oral Substudy of the Women's Interagency HIV Study Collaborative Study Group*. Effect of HAART on salivary gland function in the Women's Interagency HIV Study (WIHS). Oral Dis. 2009; 15(1): 52-60. doi: 10.1111/j.1601-0825.2008.01456.x

14 - Brooks JK, Jones JL, Price JB. Possible association of sialolithiasis with HIV infection and highly active antiretroviral therapy: A case report. Spec Care Dentist. 2020; 40(3): 298-302. doi: 10.1111/scd.12455 
15 - Arifa SP, Christopher PJ, Kumar S, Kengasubbiah S, Shenoy V. Sialolithiasis of the Submandibular Gland: Report of Cases. Cureus. 2019; 11(3): e4180. doi: 10.7759/cureus.4180

16 - Beahm DD, Peleaz L, Nuss DW, Schaitkin B, Sedlmayr JC, RiveraSerrano CM, Zanation AM, Walvekar RR. Surgical approaches to the submandibular gland: a review of literature. Int J Surg. 2009; 7(6): 503-9. doi: 10.1016/j.ijsu.2009.09.006 


\title{
Submandibular sialolithiasis in HIV-positive patient using antiretrovirals: case report
}

\begin{abstract}
Sialolithiasis is a relatively common obstructive salivary gland disorder with a uncertain pathogenesis. Since the beginning of the HAART era, studies have raised hypotheses about the increased prevalence of stones in several locations in HIV-infected patients. The present article reported a clinical case of the possible association between the formation of salivary calculus in a patient diagnosed with HIV and in treatment using antiretrovirals.

KEYWORDS: Salivary Gland Calculi; HIV; Anti-Retroviral Agents; Antiretroviral Therapy, Highly Active
\end{abstract}

\section{Como citar este artigo}

Brito LT, Oliveira VM, Paula LM; Tavares TO, Toledo IC. Sialolitíase submandibular em paciente HIV-positivo fazendo uso de antirretrovirais: relato de caso. Rev Odontol Bras Central 2021; 30(89): 290-299. DOI: 10.36065/robrac.v30i89.1474 